\title{
Inhalt / Sommaire
}

Adolf Faller, Elemente einer Wissenschaftslehre und einer Wissenschaftskritik in den Schriften von Niels Stensen (1638-1686)

Erwin H. Ackerknecht, Zur Geschichte der Krebsbehandlung 189

Beat Rüttimann, Zur Geschichte der Krüppelfürsorge 199

Albert Wirz, Malaria-Prophylaxe und kolonialer Städtebau: Fortschritt als Rückschritt? 215

Heinz G. Schott, Elemente der Selbstanalyse in den «Studien über Hysterie» 235

Johann Ulrich Marbach, Domenico Cirillo 257

Gian Töndury, Anatomie in Zürich 271

Heinrich Buess und Marie-Louise Portmann, Berühmte Schweizer Ärzte 289

Kürzere Beiträge

Marta Meyer-Salzmann, Die Spitalversorgung im Emmental 307

Heinz Balmer, Naturerkenntnis und Dichtung 314

Huldrych M. Koelbing, Pockennarben und Schönheit 321

Klaus-Dietrich Fischer, Der früheste bezeugte Augenarzt des klassischen Altertums $\quad 324$

Heinrich Buess, Paul Röthlisberger zum 70.Geburtstag 326

Berichte

Huldrych M. Koelbing, 27. Internationaler Kongreß für Geschichte der Medizin, Barcelona 1980

Huldrych M.Koelbing, Société Internationale d'Histoire de la Médecine (SIHM)

Buchbesprechungen

Hedwig Schleiffer, Narcotic Plants of the Old World (Walter Rytz)

Wolfgang-Hagen Hein und Holm-Dietmar Schwarz (Hrsg.), Deutsche Apotheker-Biographie (M-Z) (Gottfried Schramm)

Elisabeth Bennion, Antique Medical Instruments (Urs Boschung) 331

Beny Olonetzky, Die Sammlung (Urs Boschung) 333

David Nachmansohn, German-Jewish Pioneers in Science (Erwin H. Ackerknecht) 334

Hansruedi Isler (Hrsg.), Neurological Sciences in Developing countries (Erwin H. Ackerknecht)

J.M.López Piñero, F.Bujosa, M.L.Terrada, Clásicos Españoles de la Anatomía Patológica anteriores a Cajal (Erwin H. Ackerknecht)

John Cule, Wales and Medicine (Huldrych M. Koelbing)

Urs-Peter Beerli, Medizinisches in Scheuchzers «Physica sacra» oder Kupferbibel (Heinz Balmer)

Nicolas de Haller, Albert de Haller, 1708-1777 (Heinz Balmer) 339

Marta Meyer-Salzmann, Michel Schüppach, 1707-1781 (Heinz Balmer)

Berner Geographische Mitteilungen (Georges Grosjean, Aus der Geschichte des geographischen Instituts) (Heinz Balmer) 